\title{
Apuntes para una articulación crítica entre un proyecto ontológico de liberación y un programa curricular en construcción. Caso: Lic. en Filosofía e Historia de la UGC Notes for a critical articulation between an ontological liberation project and a curricular program under construction. Case: Lic. in Philosophy and History of the UGC \\ Cristhian Ricardo Santos ${ }^{1}$ \\ Universidad La Gran Colombia - Colombia
}

DOI: http://dx.doi.org/10.15648/am.32.2018.3

\begin{abstract}
Resumen
El presente artículo pretende dar a conocer los resultados y discusiones de un trabajo de investigación que se propone realizar una lectura "no eurocéntrica" de aquellos criterios, principios y conceptos que fundamentan el marco teórico-conceptual, bajo el cual se busca formar a los futuros licenciados en Filosofía e Historia de la Universidad La Gran Colombia, a partir del análisis de la documentación legal vigente que la rige y teniendo como marco de referencia el proyecto ético-pedagógico expuesto por Enrique Dussel en su obra Ética de la liberación en la época de la globalización y la exclusión publicada en 1998. Este trabajo giró en torno a la propuesta metodológica de una investigación formativa de tipo cualitativo donde el grado de profundidad desde el que se abordó el fenómeno concreto de estudio, estuvo enmarcado dentro de los límites de un nivel exploratorio de investigación y un análisis documental. El trabajo se dividió en dos partes: primero, el análisis crítico de los criterios, principios y conceptos del proyecto ético-pedagógico propuesto por Dussel y materializado en su ética de 1998 y segundo, el analisis crítico de los criterios, principios y conceptos que fundamentan el marco teórico-conceptual del programa de Licenciatura en Filosofía e Historia de la Universidad La Gran Colombia, para así, finalizar con la develación de las condiciones de posibilidad de una articulación crítica entre estos dos proyectos ontológicos.
\end{abstract}

Palabras clave: Enrique Dussel, proyecto ético-pedagógico, ética de la liberación, PEI, PEP, modelo pedagógico, Universidad La Gran Colombia.

\begin{abstract}
The present philosophical research text proposes a "No-Eurocentric" reading of those criteria, principles and concepts that underlie the theoretical framework under which it is intended to train the future graduates in Philosophy and History of Universidad La Gran Colombia, based on the analysis of the legal documentation in force that governs it and having as reference the ethical-pedagogical project presented by Enrique Dussel in his work: Ética de la liberación en la época de la globalización y la exclusion, published in 1998. So, in agreement with this objective, this work is around the methodological proposal of a formative research of qualitative type where the degree of depth from which the concrete phenomenon of study, would be addressed will be marked within the limits of an exploratory level of investigation and an analysis documentary papers. For these reasons, the present philosophical work will be divided in two parts: the first, the critical analysis of the criteria, principles and concepts of the ethical-pedagogical project proposed by Dussel and materialized in his 1998 Ethics. Second the critical analysis of the criteria, principles and concepts that underpin the theoretical framework of the undergraduate program in Philosophy and History of the Universidad La Gran Colombia, and thus, to end with the unveiling of the conditions of possibility of a critical articulation between these two ontological projects.
\end{abstract}

Keywords: Enrique Dussel, ethical-pedagogical project, ethics of liberation, PEI, PEP, pedagogical model, University La Gran Colombia.

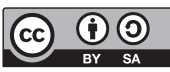

Cita de este artículo (APA): Ricardo, C. (2018). Apuntes para una articulación crítica entre un proyecto ontológico de liberación y un programa curricular en construcción. Caso: Lic. en Filosofía e Historia de la UGC. Amauta, 16(32), 31-47. http://dx.doi.org/10.15648/am.32.2018.3

1. Autor de correspondencia: Correo electrónico: cristhian.santosugc@gmail.com 


\section{Introducción}

La crítica del pensamiento latinoamericano hacia el pensamiento occidental y a su vez, en retrospectiva hacia sí mismo, se encarga de forjar como marco de referencia un objeto y objetivo de estudio que, para lo que compete aquí, radica en el reconocernos como latinoamericanos capaces de crear y reflexionar frente a las múltiples mitificaciones planteadas por el pensamiento impuesto, es decir, por el pensamiento hegemónico colonial, el cual devela, ante la necesidad de un pueblo subyugado y oprimido, las condiciones de posibilidad transformadoras, de superación, no solo ontológicas, sino también, epistemológicas.

El pensar la producción de una filosofía latinoamericana, parte de la reflexividad particular de la propuesta sobresaliente ético-política crítica emergente en la segunda mitad del siglo XX, donde Enrique Dussel, asumiendo el filosofar como un vincularse con lo circundante, planteará a la ética como filosofía primera, la cual, en sus inicios (1973) será propuesta para Latinoamérica, pero, posteriormente, en 1998, como una ética mundial con pretensión de universalidad, sopesada bajo el principio ético-material de producir, reproducir y desarrollar la vida del sujeto en comunidad (Dussel, 1998). Al respecto, dice Gonzales (2011):

Así, la ética se establece como filosofía primera, el principio sine qua non de la construcción colectiva y humana. Ya que una vez se ha dado el reconocimiento del otro como sujeto ético y las víctimas se identifican a sí mismas, como tales, descubriendo la existencia de otras como ellas, es posible todo lo demás, los acuerdos válidos intersubjetivos, afirmación analéctica, procesos de liberación, etc. (p.102)

Y en relación, afirma Díaz (2000):

Como tarea de inclusión y liberación la preocupación de la ética de la liberación apunta a la necesidad de practicar los derechos humanos y a la necesidad de sensibilizar y percibir nuevos derechos que todavía no han emergido o no han conseguido expresarse totalmente. (p.389) 
No es un secreto y para nuestros tiempos, es necesario asumir que, nos encontramos ante una crisis mundial, entendida esta desde las diferentes dimensiones que conforman al hombre y su entorno. Esto es, aquello que ha construido, como lo hizo en las crisis del siglo XX, una necesidad real de buscar diferentes alternativas a las problemáticas emergentes, y a su vez, que ha creado una insatisfacción con el sistema vigente, una toma de conciencia por parte de las comunidades afectadas y la construcción de nuevas racionalidades. Todo sistema, sea el que sea, generará víctimas, y, por ende, mientras existan, la ética de la liberación será un proyecto vigente y esencial para su comprensión, y posible solución, debido a que la filosofía, aquí, es la encargada de pensarse las realidades, pero la ética es aquella que posibilita y anima a transformarlas. Es decir: "la ética de la liberación pretende un reconocimiento humano que no se reduzca a una posición paternalista, comprometiendo cada hombre y mujer a ser parte actuante y constructora de la realidad mundial" (Freire, 2013, p.105).

En el proyecto de la ética de la liberación, entendido por unos como una ética preventiva, busca la consolidación de un humanismo universal que, bajo sus principios de reconocimiento cultural, descentralización de la razón, una racionalidad abierta a la historia, una práctica liberadora, la ciudadanía y la participación popular, la preservación del medio ambiente, la solidaridad de las naciones como práctica de la justicia y la convivencia, y a su vez, la necesidad de cambio y transformación a partir de la concientización de su ser forja unas relaciones dialécticas o pares categoriales que en el discurso dusseliano ayudará a entender su necesidad hoy (Pórtela, 2015).

Es así como Dussel, según Yori (2001), intenta analizar el funcionamiento y la posible solución de problemáticas concretas en las vidas cotidianas de las comunidades excluidas, como la expuesta anteriormente. Es evidente aquí que el sistema globalizado y la vida humana, las políticas del norte y las necesidades del sur, los dominadores y las víctimas, el fetichismo del sistema y los procesos de liberación, la ética justificadora de la totalidad o el sistema y la ética de la liberación para la vida digna de cada hombre; todos, sin excepción, posibilitan el concebir múltiples alternativas, a partir de su apropiación y concientización ética, pero, son aquellas categorías las que han potenciado 
a la ética de la liberación y su discurso como una alternativa, no única ni absoluta, pero sí necesaria para comprender la realidad latinoamericana hoy, y aquellas necesidades y realidades locales de cualquier país o comunidad excluida por el sistema vigente. Al respecto dice Yori (2001):

El discurso de la ética de la liberación pretende devolver status ontológico al discurso crítico-emancipatorio desde dos polos. En primer lugar, se apoya en la construcción del rasgo unitario del sujeto éticocorporal desde una ontología del ser vivo; $y$, en segundo lugar, se apoya en la racionalidad ético-pre-originaria y ético crítica como ejercicio ampliado de la razón. (p.154)

En este contexto, la presente investigación tuvo como objetivo proponer una lectura "no eurocéntrica", a partir del proyecto ético-pedagógico de liberación expuesto ${ }^{2}$ por Enrique Dussel en su obra Ética de la liberación en la época de la globalización y la exclusión (1998), de aquellos criterios, principios y conceptos que fundamentan el marco teórico bajo el cual se pretende formar a los futuros licenciados en Filosofía e Historia de la Universidad La Gran Colombia, donde, fue necesario enmarcar la discusión tanto en el ámbito de la ética y sus fundamentos, como en el de la pedagógica, entendida esta como aquella mediación práctica que, sitúa su espacio de acción en la vida misma, en otras palabras, en donde se producen las relaciones de dominación.

Para esto, los esfuerzos se situaron, primero, en la exposición y reconocimiento del origen y razón de ser de la ética de la liberación partiendo, claro está, del problema de la autenticidad de una "filosofía latinoamericana" y a su vez de cómo se posibilitó el tránsito hacia una filosofía de la liberación o primera ética dusseliana. La ética de la liberación tiene como marco de reflexión y andamiaje teórico a la filosofía de la liberación, por lo que fue indispensable, para una comprensión arquitectónica de la segunda ética dusseliana, reconocer el debate que, en la segunda mitad del siglo XX, se gesta

2. En el presente texto se emplearán algunas abreviaturas para facilitar su lectura, las cuales son presentadas a continuación: Proyecto Ético-Pedagógico de Liberación (PEPL), Universidad La Gran Colombia (UGC), Programa Educativo Institucional (PEI), Proyecto Educativo del Programa (PEP), Programa de Licenciatura en Filosofía e Historia (PLFH), Modelo Pedagógico Institucional (MPI). 
a partir de sus postulados. Ya, por último, asumiendo la ética de la liberación como alternativa filosófica y una propuesta construida desde una perspectiva latinoamericana se intentó mostrar su importancia frente al debate ético contemporáneo.

Posteriormente, enfocamos nuestro análisis en las categorías conceptuales que, posibilitarían una comprensión arqueológica del proyecto éticopedagógico de liberación, propuesto por Enrique Dussel en su obra de 1998 anteriormente referida. Ya por último, se hizo hincapié en la necesidad de identificar los criterios, principios y conceptos que fundamentan el programa de Licenciatura en Filosofía e Historia de la Universidad La Gran Colombia, a partir de la revisión documental del modelo pedagógico, el PEI y el PEP de la institución; para así, ya por último, poder determinar las condiciones de posibilidad para una articulación crítica entre el proyecto ético-pedagógico de liberación, propuesto por Enrique Dussel en su obra anteriormente enunciada y el marco teórico-conceptual consignado dentro de la documentación legal vigente del programa de Licenciatura en Filosofía e Historia de la Universidad La Gran Colombia.

A continuación, se presentan los fundamentos metodológicos de una investigación formativa y su pertinencia con el trabajo, según el CNA y Osorio (2008); seguidamente el diseño y nivel de investigación (Arias, 1999), los elementos que constituyeron la metodología, el tratamiento de la información y los resultados.

\section{Metodología}

El presente trabajo se encuentra adjunto a una de las tres líneas primarias de investigación propuestas por la UGC, denominada "pensamiento socio crítico para la construcción significativa y solidaria del conocimiento", la cual busca, que a partir de la construcción de un marco teórico, conceptual y práctico se analicen las diferentes problemáticas que presenta la educación en Colombia y su incidencia sobre cada una de las disciplinas de formación académica presentes en la Facultad de Educación de la misma universidad. Tras esto y con el fin de contribuir a la construcción de pensamiento crítico-reflexivo 
concreto se propuso una ruta de trabajo, soportada dentro del marco de una investigación formativa, la cual está sustentada bajo una racionalidad crítica, debido a que, esta asume condiciones de objetividad, subjetividad e intersubjetividad, no solo desde factores epistemológicos, sino también desde aquellos factores históricos que generen una correlación entre sí posibilitando una coherencia entre teoría-práctica y una potenciación de las múltiples dimensiones que afectan una cultura investigativa. Al respecto, Osorio (2008) dice

...la investigación formativa es entendida como proceso problémico, intencional e interdisciplinario, cuyo eje medular son los métodos de las ciencias humanas y/o naturales que propendan por el empoderamiento del maestro/a en formación y del/la docente a partir de su inserción en una cultura de la investigación en las que ambos desarrollen competencias comunicativas e investigativas con miras a la cualificación y transformación de su práctica pedagógica. (p.48)

Es por esto que, dentro de la investigación formativa y la polisemia de dichos términos, su utilización en cuanto a enfoques metodológicos está determinada por el énfasis del trabajo y la intencionalidad del investigador frente al objeto de estudio de la investigación. En este sentido, siguiendo a Osorio (2008) es necesario aclarar, que la investigación formativa intrínsecamente se solventa bajo tres énfasis orientadores, los cuales son: la investigación exploratoria, la formación en y para la investigación y por último la investigación para la transformación en la acción o práctica.

El primer énfasis enunciado anteriormente, es decir, la investigación exploratoria, fue el andamiaje metodológico general del trabajo en mención y sobre el cual se determinarán los niveles de investigación, el diseño de la investigación, sus fases, técnicas e instrumentos con miras al análisis del objeto de estudio; en nuestro caso el Programa de Licenciatura en Filosofía e Historia de la UGC. Al respecto dice Osorio (2008):

En primer lugar, está la investigación exploratoria, propia de los métodos y técnicas de la investigación científica, esta cobra importancia 
cuando no existe claridad acerca del problema o proyecto por ausencia de información porque permite indagar y explorar necesidades, problemas, hipótesis y poblaciones; adicionalmente, aporta información que posibilita construir interrogantes y enfoques más precisos. Uno de sus propósitos fundamentales es dar estructura lógica y metodológica a un proyecto de investigación. Por tanto, acá la investigación asume un enfoque "formativo" es decir, se trata de la investigación en sí, no de la formación del estudiante. (pp.46-47)

Teniendo en cuenta esto, es evidente la necesidad de una investigación formativa en un trabajo de tipo cualitativo como el que se realizó aquí, dadas las pretensiones de este frente a la construcción del conocimiento.

El nivel de investigación, definido por Arias, como "el grado de profundidad con que se aborda un objeto o fenómeno" (1999, p. 19), nos permitió clasificar, frente a sus tres niveles propuestos (investigación exploratoria, investigación descriptiva, investigación explicativa), el presente trabajo dentro una investigación exploratoria, la cual es caracterizada por un tema u objeto poco conocido y estudiado, y a su vez por unos resultados encaminados a una visión aproximada de dicho objeto de estudio.

En consecuencia, con lo anterior, el diseño de investigación, entendido aquí, según Arias (1999) como: "la estrategia que adopta el investigador para dar respuesta al problema planteado" (p.21), tuvo en cuenta la investigación documental, la cual tiene como base la obtención y análisis de datos incorporados en materiales impresos u otro tipo de documentos. Dicha recolección de información se realizó a partir de técnicas propias de una investigación exploratoria y documental, como el fichaje, el Resumen Analítico de Investigación (RAI), el análisis de contenido, la toma de apuntes, entre otros. Y a su vez, teniendo como instrumentos esenciales fichas, libretas y guías para el análisis de contenido.

\section{Triangulación de la información}

Los tres momentos expuestos a continuación resumen tanto el proceso de revisión bibliográfica necesario para el desarrollo del proyecto en mención 
como la discusión emergente a partir de dicha lectura, no solo con aquellos hechos histórico-culturales, socioeconómicos y ético-políticos paralelos al proceso de consolidación de una filosofía latinoamericana, o, bajo nuestros intereses, de una filosofía y ética de la liberación dusseliana a nivel mundial, sino también de aquellos argumentos expuestos por ciertos de pensadores del NORTE y a su vez del SUR, detractores de esa propuesta anti-hegemónica, anti-capitalista, centrada en las víctimas del sistema vigente y en toda posibilidad de transformación de su condición.

a) La pregunta por el origen y razón de ser de la ética de la liberación, como primer momento investigativo, nos remontó a la discusión en torno al problema de la autenticidad de una "filosofía latinoamericana" y a su vez, de cómo esta posibilitó el tránsito hacia una filosofía de la liberación o primera ética dusseliana. Posteriormente y entendiendo a la filosofía de la liberación como marco de reflexión y andamiaje teórico para la consolidación de una ética de la liberación fue necesario realizar un acercamiento al debate que en la segunda mitad del sigloXX se gesta a partir de sus postulados. Ya por último y asumiendo la ética de la liberación como alternativa filosófica se intentó mostrar su importancia frente al debate ético contemporáneo.

Siguiendo a Álvarez (2007) y a Dussel (1994) la división del proceso de consolidación de la ética de la liberación la podemos sintetizar, teniendo en cuenta los avatares histórico-culturales del contexto bajo el cual se constituye, en tres premisas fundamentales que son:

- Primero, que la filosofía latinoamericana, vista y entendida desde su gestación se hizo gracias a la "imitación" y el no reconocimiento activo de su ser ante la enajenación para con la filosofía tradicional occidental.

- Segundo, que la Filosofía de Liberación, se constituye a partir de la superación de los dogmatismos moderno-europeos impuestos en Latinoamérica, priorizando los procesos de la institucionalización de la filosofía.

- Tercero, que la Ética de la Liberación propiamente dicha, estuvo, está y estará caracterizada por el reconocimiento y reflexión crítica sobre las necesidades de América Latina a partir de la adopción de un lenguaje liberador político-humanista frente a los procesos de globalización y exclusión del sistema hegemónico vigente. 
Dichas premisas contribuyeron a la discusión desde dos flancos teniendo en cuenta a la filosofía y a la ética misma, ya que, la filosofía entendida como producción cultural, según Dussel (1996), es considerada como una forma para comprender la realidad, pero aún más importante, para transformarla. Es decir que, la filosofía y su quehacer no es simplemente el filosofar sobre otras filosofías; filosofar abstrayéndose como no lo ha heredado la tradición moderna, sino una filosofía de lo concreto y de la praxis donde el filósofo se ha adentrado en aquella aventura teórico-práctica, la cual ha partido de la realidad para autoservirse, criticarse y transformarse.

Por otro lado, la ética entendida como filosofía primera se propone hoy como una alternativa viable para comprender, primero, el sufrimiento de las mayorías pobres de la humanidad; después criticar las lógicas económicas, políticas, éticas, pedagógicas, entre otras, propuestas por la globalización, las cuales posibilitan dicho sufrimiento, y segundo, transformarlas, ya que, como quehacer ético-filosófico propiamente dicho la posibilidad de unión de la crítica, que parte de las víctimas, con la creación o creatividad, principio originario de la transformación de su situación, posibilitan un responsabilizarse materialmente por el otro.

b) La Ética de la liberación asume aquí, como requerimientos conceptuales, unos principios bajo los cuales, según Dussel (1998), debe fundamentarse toda ética. La ética I o fundamental asume dentro de sí tres grandes momentos o núcleos problémicos: 1) la verdad práctica, 2) la validez intersubjetiva y 3 ) la factibilidad. Cada uno de estos momentos, constitutivamente, subsume un criterio o condición de posibilidad que, dentro de la realidad objetiva y sus relaciones dadas, construidas y re-construidas de contenido, funciona como herramienta y unidad de medida ética; un principio o fundamento que subsume aquellas condiciones de posibilidad materiales, formales-instrumentales (validez) y de factibilidad como momentos constitutivos de una ética material.

La ética I o fundamental es necesaria pero no suficiente para el análisis y transformación de un contexto histórico-cultural, socio-político y económico actual concreto. América Latina padece hoy, al igual que Asia, África y los sectores oprimidos del centro, la exclusión como mayoría de la humanidad, 
sobreviviendo así bajo una situación de vulnerabilidad social intra-sistémica; ya que conforma el mundo periférico, pero aún más importante, se desenvuelve en una realidad concreta que niega la propia vida. La ética II o criticidad ética es el complemento necesario para una total aplicabilidad de los momentos fundantes, anteriormente nombrados, de una ética fundamental.

Ahora, esta parte de la Ética de la liberación asume como problema central la negación de los principios de la Ética I o fundamental y como punto de partida la negación de la vida, su opresión y exclusión.

A continuación es imprescindible realizar algunas precisiones conceptuales posibilitadoras, primero, de una comprensión arquitectónica de la ética de la liberación y segundo, de un análisis crítico de aquellos criterios y principios que fundamentan el proyecto ético-político de liberación propuesto por Enrique Dussel.

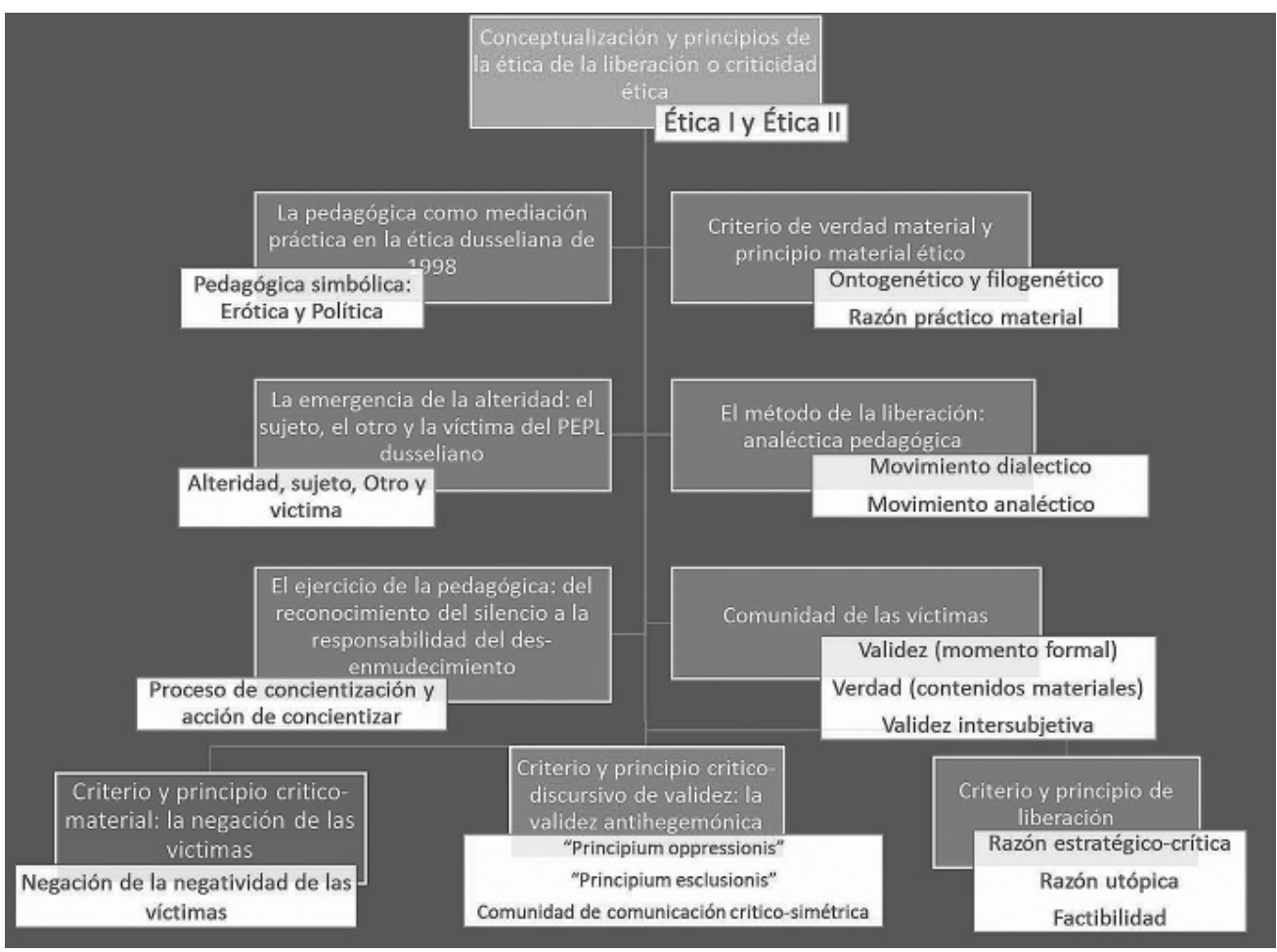

Figura 1. Marco teórico-conceptual: PEPL propuesto por Enrique Dussel 
En la Figura 1 se refleja el acercamiento que se realizó a la obra de Enrique Dussel ya referenciada donde se caracterizaron cada uno de los seis principios expuestos entre la primera y segunda parte de la obra, junto con sus criterios negativos y positivos, bajo el marco de la categoría de pedagógica desde el cual se comenzó a entablar el diálogo entre universidad y ética de la liberación a partir de la asimilación de la categoría de diálogo de saberes o diálogo generacional y praxis de liberación, entendida esta como aquella práctica crítica que asume sujetos históricos, no desde el principio de imponer al Otro, sino de escuchar la voz del Otro. En efecto, se devela la pretensión del proyecto ético-pedagógico dusseliano, esto es la consolidación, a partir de la Ética de la liberación, de un humanismo con pretensión de universalidad.

c) Pensar en retrospectiva la universidad fue un momento clave de esta investigación, el cual sirvió para identificar la fundamentación ético-humanista de la UGC que hasta el momento la había orientado en cuanto a la formación de sus profesionales. Es por esto que volver a su origen nos remitió a pensar en las causas y antecedentes de su emerger, el contexto bajo el cual surgió y cuáles fueron los insumos teórico-conceptuales que impulsaron dicho surgimiento. Seguido de esto, fue importante identificar y analizar aquellos principios fundacionales y filosóficos, que junto a los criterios misionales de la UGC, forjaron un proyecto ontológico éticohumanista expuesto en su PEl. También se intentó comprender los diferentes enfoques y dimensiones, como lo son lo histórico-cultural, antropo-sociológico y socio-comunitario desarrollados en el MPI y su debida articulación con los criterios y principios propuestos por el PEI del PLFH.

Es aquí, donde a partir de una lectura no eurocéntrica, se intentó alentar el debate contemporáneo en torno a la ética de la liberación como proyecto ontológico alternativo al hoy predominante y a la pedagógica como mediación práctica de transformación. La siguiente figura demarca los criterios ético-humanistas propuestos por la UGC y, por ende, materializado en el programa de Licenciatura en Filosofía e Historia. 


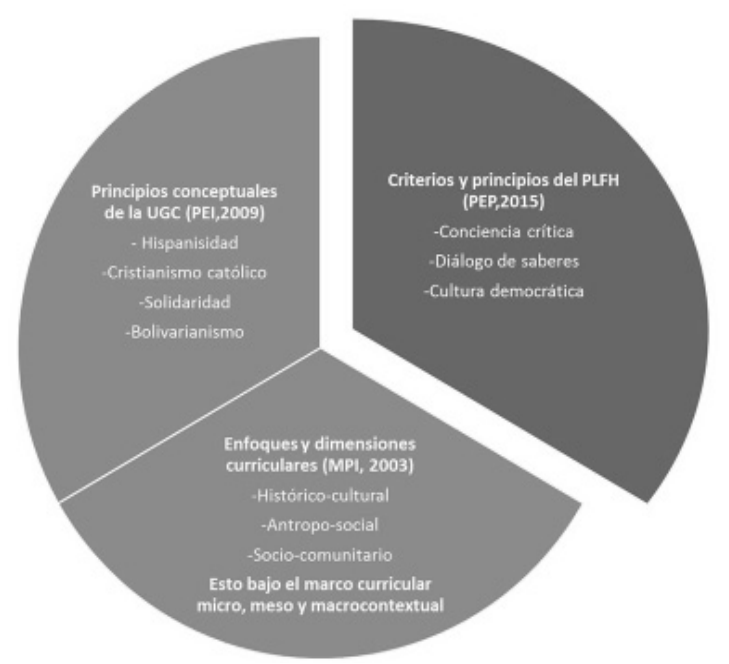

Figura 2. Criterios y principios del proyecto ético-humanista del PLFH de la UGC

Según estos criterios y principios reflejados en la Figura 2, el marco teóricoconceptual desde el que se pretende formar aquellos sujetos históricos encargados de la transformación del proyecto ontológico de dominación actual no es suficiente, ya que el vehículo posibilitador de que dichos planteamientos tomen un sentido, es decir el MPI, aún se encuentra cimentado bajo los paradigmas universales de los procesos de enseñanza-aprendizaje, mas no desde esas problemáticas cotidianas y particulares que determinan la posibilidad de una transformación real de un proyecto ontológico.

Es un modelo que asume un discurso ajeno a su realidad concreta, ya que sus fundamentos teóricos han sido recepcionados acríticamente; dicho de otra manera, han sido planteamientos que, sin repensarse, según las necesidades contextuales de la población, fueron implementados y apropiados como verdad unívoca y absoluta. En efecto, el MPI como mediación práctico-cultural debe corresponder no solo con la realidad de las víctimas, la cual pretende transformar, sino también con aquel proyecto ético-político propuesto en el PEl; problema que aquí nos comienza a ser evidente.

Para poder comenzar a generar alternativas frente a dicha problemática se realizó una lectura comparada bajo el análisis categorial, de contenido, señalado a continuación: 


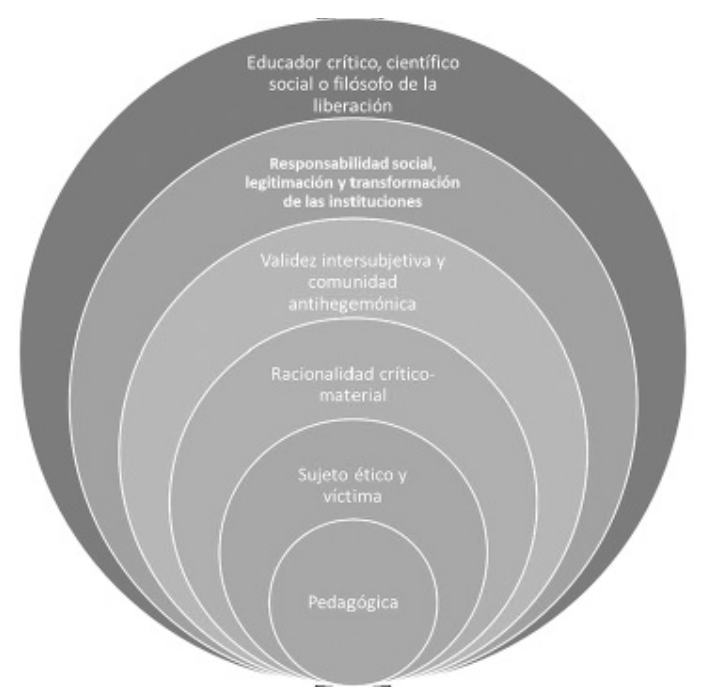

Figura 3. Marco categorial: análisis comparativo entre dos proyectos ontológicos

A partir de este análisis se nos hace evidente que lo pedagógico es la mediación práctica por excelencia de todo proceso ético-político de formación y que su fundamento son las relaciones dialécticas. No obstante, es aquí donde la pedagogía deja de ser simplemente aquella ciencia de la enseñanza y el aprendizaje, para convertirse en la parte de la filosofía encargada del pensarse esa relación cara-a-cara entre maestro-discípulo, político-ciudadano, padre-hijo, entre otros. Es decir, para convertirse en lo que Dussel va a denominar como pedagógica.

Bajo el marco teórico-conceptual propuesto desde un proyecto ético-pedagógico de liberación toman sentido los interrogantes ¿Para qué enseñar y para qué aprender si los fines educativos o los mismos propósitos formativos están cimentados en unas relaciones asimétricas de dominación donde lo que se busca es la supresión de toda subjetividad Otra? ¿Qué saber? Cuando se privilegian aquellas teorías pedagógicas, políticas, filosóficas, científicas, sociales, entre otras, que responden a unas situaciones histórico-culturales distintas a las construidas en Latinoamérica. ¿Cómo enseñar y cómo aprender? Si las estructuras y organizaciones curriculares, estrategias metodológicas y didácticas se fundamentan en una relación dialéctico-dominadora donde el cara-a-cara del maestro-discípulo parte, no desde el principio de escuchar al otro, sino de imponer al otro, negarlo y declararlo causa culpable 
de su negación. ¿Hasta dónde se aprendió? Es decir, qué tanto pueden reproducir o no las relaciones asimétricas de dominación propuestas desde los fines educativos.

\section{A modo de conclusión}

El propósito del presente trabajo filosófico de investigación concentró sus esfuerzos en dar respuesta al interrogante ¿Por qué el proyecto ético-pedagógico de liberación expuesto por Enrique Dussel en su obra Ética de la liberación en la época de la globalización y la exclusión (1998) posibilita una lectura "no eurocéntrica" de aquellos criterios, principios y conceptos que fundamentan el marco teórico, bajo el cual se pretende formar a los futuros licenciados en Filosofía e Historia de la Universidad La Gran Colombia? Los argumentos aquí expuestos pretendieron siempre posibilitar la comprensión del proyecto ético-pedagógico desde una arquitectónica dusseliana, donde, como pretensión general no se buscaba abarcar, ni mucho menos agotar la riqueza temática y conceptual que contiene la obra del pensador mendocino Enrique Dussel. Esto es tan solo un acercamiento interpretativo que, busca, más que situarse como verdad absoluta y unívoca, abrirse ante la discusión y retroalimentación académica de profesores, estudiantes y todos aquellos sujetos históricos que se ponen al servicio de la vida humana (Vega, 2015).

Lo anterior nos conlleva a señalar, de forma clara y precisa, aquellos aspectos que contribuyeron al desarrollo del interrogante anteriormente nombrado y a su vez, a los resultados obtenidos a partir de la ruta seleccionada desde el inicio de la presente investigación.

- La construcción del pensamiento filosófico latinoamericano, la filosofía de la liberación y la ética de la liberación son el esfuerzo mancomunado de un sinnúmero de pensadores, que desde Mariátegui hasta Dussel, enmarcados por sus avatares y por aquellas discusiones intraculturales e interculturales, posibilitaron que, desde una historia otra, desde una sabiduría popular, un ethos antropológico y una reflexión concreta la búsqueda de la identidad desde y para América se convirtiera en aquel horizonte de sentido y objeto de reflexión crítico-material. 
- La pedagógica como posibilidad de un diálogo entre generaciones y una praxis de liberación es entendida como aquella práctica crítica que asume sujetos históricos, no desde el principio de imponer al Otro, sino de escuchar la voz del Otro; no es negar al Otro declarándolo causa culpable de su negación (el mito de la modernidad), sino, es negar la negación producida por la pedagógica de la dominación. Es un deseducarse y reeducarse.

- La Ética de la liberación busca la consolidación de un humanismo universal que, bajo sus principios de reconocimiento cultural, descentralización de la razón, una racionalidad abierta a la historia, una práctica liberadora, la ciudadanía y la participación popular, la preservación del medio ambiente, la solidaridad de las naciones como práctica de la justicia y la convivencia, y a su vez, la necesidad de cambio y transformación a partir de la concientización de su ser constituirán a la ética como filosofía primera y a la vida como condición límite de toda acción humana. Es decir, que la ética está sopesada bajo el principio ético-material universal de producir, reproducir y desarrollar la vida del sujeto en comunidad (Dussel, 1998).

- Las condiciones de posibilidad para la articulación crítica entre el PEPL propuesto por Dussel en su obra Ética de la liberación en la época de la globalización y la exclusión (1998) y el PLFH, funda su viabilidad bajo el marco de asimilación de la dimensión y el enfoque pedagógico como mediación práctica y punto de convergencia entre la adquisición y producción del conocimiento y la construcción de nuevos sujetos históricos; pero sobre todo, en cuanto a la formación de educadores crítico-solidarios, asumiendo la relación entre el aprender como transformación y la escucha como apertura a la reflexión crítica.

- La inevitable generación de víctimas por parte de todo sistema ético-político justifica la crítica, no solo al sistema como totalidad, sino, a cada una de sus instituciones político-pedagógicas encargadas de la formación de sujetos históricos. La UGC y no solo el PLFH, deberán partir del hecho de reconocer la necesidad de resignificar los criterios y principios que fundamentan la razón de ser y hacer de la misma, como institución políticopedagógica antihegemónica y del profesional crítico como ser humano autorresponsable y corresponsable por la producción, reproducción y desarrollo de la vida humana de los sujetos en comunidad (Dussel, 1998). 
Este trabajo es la apertura o primer acercamiento a la posibilidad de una articulación crítica, no solo por la develación de los fundamentos de ambos proyectos ontológicos, sino también por la pretensión y promoción de una lectura comparada a la luz de las necesidades contextuales del PLFH.

Esto es tan solo una interpretación que se propone para ser debatida y retroalimentada, que como reflexión filosófica da la apertura; apenas abre el camino a un tema que tiene mucho por indagar.

\section{Referencias bibliográficas}

Acevedo, R. (2007). Fundamentación y vigencia de la ética de la liberación en Enrique Dussel. Bogotá: Universidad Nacional de Colombia. Facultad de Filosofía.

Álvarez, S. (2007). La liberación como proyecto ético: Un análisis a la obra de Enrique Dussel. Madrid: Universidad Complutense de Madrid. Facultad de Filosofía.

Apel, K.-O. (2005). Ética del discurso y ética de la liberación. México: Trotta.

Arias, F. (1999). El Proyecto de investigación: guía para su elaboración. Caracas: Episteme.

Díaz, G. (2000, 2001). Enrique Dussel en la filosofía latinoamericana frente a la filosofía eurocéntrica. Valladolid: Universidad de Valladolid. Facultad de Filosofía.

Dussel, E. (1980). La pedagógica latinoamericana. Bogotá: Nueva América.

Dussel, E. (1994). 1492 El encubrimiento del otro. Hacia el origen del "mito de la modernidad". Bolivia: Plural Editores.

Dussel, E. (1994). Historia de la filosofía latinoamericana y filosofía de la liberación. Bogotá: Editorial Nueva América.

Dussel, E. (1996). Filosofía de la liberación. Bogotá: Nueva América.

Dussel, E. (1998). Ética de la liberación en la edad de la globalización y la exclusión. México:Trotta.

Dussel, E. (2004). http://enriquedussel.com/Home_cas.html. Obtenido de http://enriquedussel.com/Home_cas.html - http://red.pucp.edu.pe/ wp-content/uploads/biblioteca/og0514.pdf

Freire, A. (2013). Ética, un camino hacia la liberación. "Análisis de la ética de la 
liberación en Enrique Dussel". Cuenca: Universidad de Cuenca. Facultad de Filosofía.

García, M. (2009). Julio César García Valencia y su participación en la educación nocturna en Colombia: desarrollo histórico y reglamentación. Bogotá: Universidad La Gran Colombia.

Gómez, T. (2008). Aporte del pensamiento bolivariano a la educación éticoliberadora de la Universidad La Gran Colombia. Bogotá: Universidad La Gran Colombia.

Gonzáles, L. (2011). Ética de la liberación: una propuesta ético crítica desde y para nuestra América. Santiago, Chile: Universidad de Chile.

Llano, F., Osorio, A., Santamaría, J., \& Osorio, R. (2012). Entre lo deseable y lo posible. Bogotá: Universidad La Gran Colombia.

Osorio, M. (2008). La investigación formativa, la posibilidad de generar cultura investigativa en la educación superior: el caso de la práctica pedagógica de la licenciatura en educación básica con énfasis en humanidades, lengua castellana de la Universidad de Antioquia. Medellín, Colombia: Universidad de Antioquia.

Pórtela, J. G. (2015). Construcción del consenso moral del consenso y ley natural. Justicia, 20(28). https://doi.org/10.17081/just.20.28.1033

Roig, A. (1993). Rostro y filosofía de América Latina. Mendoza: EDIUNC.

Sánchez, W. (2007). La universidad sin órganos. Capitalismo cognitivo y transformaciones empresariales de la universidad colombiana. Nómadas, 34-46.

Serrano, A. (1994). El doble rostro de la posmodernidad. San José: CSUCA.

Universidad La Gran Colombia (2003). Modelo Pedagógico Institucional. Bogotá: Oficina de Publicaciones.

Universidad La Gran Colombia (2009). Programa Educativo Institucional. Bogotá: Oficina de Publicaciones.

Universidad La Gran Colombia (2015). Proyecto Educativo del Programa. Bogotá: sin publicar.

Vega Arrieta, H. (2015). Aspectos dogmáticos y políticos criminales de la estructura general del delito en el sistema penal colombiano. Justicia, 20(27). https://doi.org/10.17081/just.20.27.806

Yori, P. (2001). La ética de la liberación de Enrique Dussel en el debate contemporáneo: Una lectura desde las bases teóricas y metodológicas de la historia de las ideas latinoamericanas. Mendoza: Universidad Nacional de Cuyo. Facultad de Filosofía y Letras. 